\title{
Spin Diffusion and the Role of Screening Effects in Semiconductors
}

\author{
J. Ghosh, V. Sverdlov, and S. Selberherr \\ Institute for Microelectronics, TU Wien, Gußhausstraße 27-29/E360, A-1040 Wien, Austria \\ Email: $\{$ ghosh | sverdlov | selberherr\}@iue.tuwien.ac.at
}

\begin{abstract}
The electron spin properties in semiconductors are of great interest because of their potential in future spin-driven microelectronic devices. We performed simulations on electron spin injection in an $n$-doped silicon bar from a magnetic semiconductor with spin-dependent conductivity. A variation of doping can introduce charge accumulation/depletion layer at the interface. We found substantial spin transport differences, when spins are introduced through a charge accumulated and a charge depleted interface layer. Special attention is paid to a possible spin injection efficiency enhancement compared to the existing theory at the charge neutrality condition. We found that at a fixed bulk spin polarization, the efficiency increases, if spins are injected from a charge depleted source.

Index Terms-Spin transport, spin injection, space-charge region.
\end{abstract}

\section{INTRODUCTION}

Spin injection into semiconductors by purely electrical means is paramount for building spintronic devices [1]. One of the methods is to inject spins from a ferromagnetic electrode. Recently, a robust spin injection from a ferromagnetic metal contact into a semiconductor has been performed at room temperature [2]. However, the magnitude of the spin accumulation signal obtained with a three-terminal measurement setup is several orders of magnitude larger than that predicted by theory [2]. Recently, an explanation based on the assumption that the resonant tunneling magnetoresistance effect and not the spin accumulation causes the electrically dependent spin signal in local three-terminal detection experiments, was proposed [3]. Alternatively, an evidence that accounting for the space charge effects at the interface may boost the spin injection by an order of magnitude was presented [4]. However when a charge accumulation is created at a boundary

978-1-4799-5433-9/14/\$31.00 (C) 2014 IEEE of the semiconductor, the spin current increases only close to that boundary, while at a distance of about the Debye screening length $\left(\lambda_{D}\right)$ away from the boundary, the value of the spin current is similar to the one at the charge neutrality condition, provided the spin polarization is the same [5]. A possible source of this discrepancy is that the ferromagnetic contact was not considered in [5]. We include the ferromagnetic contact and investigate the influence of the space charge effects at the ferromagnetic/non-magnetic semiconductor interface on the spin injection into a semiconductor.

Our structure is composed of a semiconductor ferromagnet $(F M,-D L<x<0)$ and silicon $(\mathrm{Si}$, $0<x<D L)$ sharing a junction. The density of states in both materials is considered similar to avoid the impedance mismatch problem. We first extend the analytical approach [6] to incorporate a semiconductor ferromagnet $(F M)$ contact and investigate the spin injection in $\mathrm{Si}$. We hereby assume $F M$ to be doped to a concentration proportionally with a factor $K_{1}$ to the doping value in the semiconductor. When the charge current flows through the junction, the spin accumulation in the semiconductor appears. We make a comparison among the analytically and the numerically simulated results, assuming $K_{1}=1$ (the charge neutrality). In the next steps, we violate the charge neutrality by varying $K_{1}$ and look for the spin injection efficiency. Special notice is paid to any enhancement/reduction of the spin current density at the interface and bulk. A conclusion is drawn at the end regarding the spin injection efficiency.

\section{MODEL}

We express the up(down)-spin concentration as $n_{\uparrow}\left(n_{\downarrow}\right)$, and the corresponding spin current as 


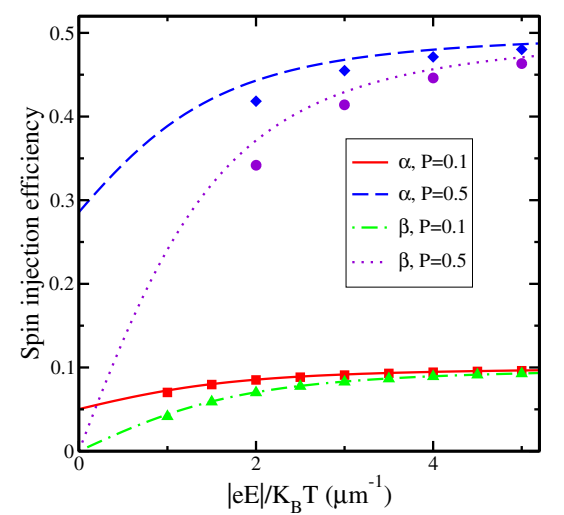

Fig. 1. Current $(\alpha)$ and density $(\beta)$ spin injection efficiencies at the $\mathrm{Si}$ interface with the applied electric field. Lines $\rightarrow$ theory (4), (5), and dots $\rightarrow$ simulation.

$J_{\uparrow}\left(J_{\downarrow}\right)$. The spin drift-diffusion equation [6], [7] and the Poisson equation are solved selfconsistently to obtain the spin concentrations $\left(n_{\uparrow}\right.$, $\left.n_{\downarrow}\right)$, and the electric potential $(U)$. The electron (spin) concentration is written as $n(s)=n_{\uparrow} \pm n_{\downarrow}$, and the electron (spin) current as $J_{n}\left(J_{s}\right)=$ $J_{\uparrow} \pm J_{\downarrow} \cdot P=\frac{s}{n}$ symbolizes the bulk equilibrium spin polarization in $F M$. Considering charge neutrality, $n=N_{D}$ (doping concentration), we express the equilibrium spin concentration $n_{\uparrow}^{e q l}\left(n_{\downarrow}^{e q l}\right)=0.5(1 \pm P) N_{D}$ (in $F M$ ) and $n_{\uparrow}^{e q l}\left(n_{\downarrow}^{e q l}\right)=0.5 N_{D}$ (in $\mathrm{Si}$ ). The respective spin deviation is $\delta n_{\uparrow}\left(\delta n_{\downarrow}\right)=n_{\uparrow}\left(n_{\downarrow}\right)-n_{\uparrow}^{e q l}\left(n_{\downarrow}^{e q l}\right)$ and $\delta s=\delta n_{\uparrow}-\delta n_{\downarrow}$.

The up(down)-spin current density can be written as [6]:

$$
J_{\uparrow(\downarrow)}=e n_{\uparrow(\downarrow)} \mu E+e D \frac{d\left(\delta n_{\uparrow(\downarrow)}\right)}{d x}
$$

$D$ is the electron diffusion coefficient, $\mu$ is the electron mobility, $E$ is the electric field, and $e$ is the elementary charge. The steady-state continuity equation for the up(down)-spin electrons including the spin relaxation reveals [6]: $\frac{d J_{\uparrow(\downarrow)}}{d x}=$ $\pm e\left(\frac{\delta n_{\uparrow}-\delta n_{\downarrow}}{\tau_{s}}\right)$, where $\tau_{s}$ is the spin life time. The Poisson equation, defining the electric field, reads: $\frac{d E}{d x}=-e \frac{n_{\uparrow}+n_{\downarrow}-N_{D}}{\epsilon_{S i}}$, where $\epsilon_{S i}$ is the electric permittivity of silicon. We denote $V_{T}$ as the thermal voltage: $V_{T}=\frac{K_{B} T}{e}$, where $K_{B}$ is the Boltzmann constant, $T$ is the temperature $(T=300 K)$. The intrinsic spin diffusion length is denoted as $L_{i}$ [6] and the diffusion

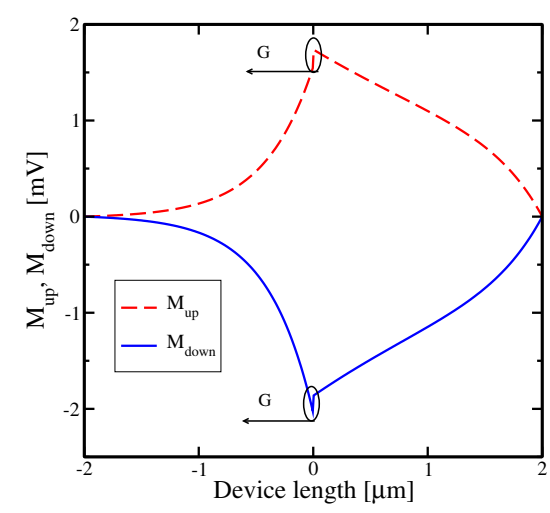

Fig. 2. A plot of $M_{u p}=V_{T} \ln \left(\frac{n_{\uparrow}}{n_{\uparrow}^{\text {eql }}}\right)$ and $M_{\text {down }}=$ $V_{T} \ln \left(\frac{n_{\downarrow}}{n_{\downarrow}^{e q l}}\right)$ through the $\operatorname{bar}\left(P=0.1, \frac{|e E|}{K_{B} T}=2 \mu \mathrm{m}^{-1}\right)$, showing a discontinuity at the junction, which gives the term $G$ in (3).

coefficient $D$ is related to the mobility by the Einstein relation $D=\mu V_{T}$. We further define the interface spin injection efficiencies for the current $\left(\alpha=\frac{J_{s}}{J_{n}}\right)$ and for the density $\left(\beta=\frac{s}{n}\right)$. The electric field dependent up(down)-spin diffusion length is denoted as $l_{u}\left(l_{d}\right)$ [6]. The up(down)-spin chemical potential is expressed as $\mu_{\uparrow(\downarrow)}$.

The above mentioned equations must be supplemented with appropriate boundary conditions. To solve the spin equations analytically, we assume the general solution for the spin density according to our convenience:

$$
\begin{array}{r}
\delta s^{F M[S i]}=\left(a_{1}\left[b_{1}\right]\right) N_{D} \exp \left(\frac{-x}{l_{d}}\right)+ \\
\left(a_{2}\left[b_{2}\right]\right) N_{D} \exp \left(\frac{x}{l_{u}}\right)
\end{array}
$$

Along with this assumption, we can set 3 boundary conditions [6]:

- $J_{\uparrow(\downarrow)}$ is continuous at the interface.

- $\delta s$ is zero at the boundaries.

- $\mu_{\uparrow(\downarrow)}$ is continuous at the interface, hence $\mu_{\uparrow(\downarrow)}^{0-}=\mu_{\uparrow(\downarrow)}^{0+}$. One can write [6]:

$$
\ln \left(\frac{n_{\uparrow(\downarrow)}^{0-}}{0.5(1 \pm P) N_{D}}\right)=\ln \left(\frac{n_{\uparrow(\downarrow)}^{0+}}{0.5 N_{D}}\right)-\frac{G}{V_{T}}
$$

$G$ is described as the spin chemical potential drop, and is responsible for spin injection from the $F M$ to the $\mathrm{Si}$ side. 
To obtain the analytical expressions, one solves for 5 unknowns $\left(a_{1}, a_{2}, b_{1}, b_{2}, G\right)$ from the above mentioned conditions. The analytical expressions at the $\mathrm{Si}$ interface for $\alpha$ and $\beta$ are cumbersome. The simplified expressions, valid for lower values of $P$, are written as:

$$
\begin{gathered}
\alpha=P \frac{l_{d}}{l_{d}+\left(1-P^{2}\right) l_{u}} \\
\beta=\left(1-\frac{l_{u}}{l_{d}}\right) \alpha \\
G=V_{T} \ln (1+\beta P)
\end{gathered}
$$

\section{Simulation AND Results}

Simulations were performed with the finite volume method (FVM) [8] for an $n$ semiconductor bar with $L_{i}=1 \mu \mathrm{m}$. A bar length of several microns, a doping concentration of $N_{D}=10^{16} \mathrm{~cm}^{-3}$, and an electron mobility of $1400 \mathrm{~cm}^{2} \mathrm{~V}^{-1} \mathrm{~s}^{-1}$ were assumed.

\section{A. Charge Neutrality}

(5) indicates that $\alpha$ is always larger than $\beta$ irrespective of the value of the applied field, and both of them tend to reach a saturation value fixed by the bulk spin polarization $(P)$ at the $F M$ side. When there is no flow of charge (charge current, $J_{c}=0$ ) from $F M$ to $\mathrm{Si}$, there is no spin injection $\left(s=0, J_{s}=0\right)$ and hence $\beta=0$. If the electric field is very small $(E \rightarrow 0), l_{u}$ and $l_{d}$ tend to $L_{i}$ and hence from (4), $\alpha \rightarrow \frac{P}{2-P^{2}}$. It is also noticed that $G$ increases with $E$ to a saturation determined by $P$. We compare our simulation results $\left(K_{1}=1\right)$ to the analytical expressions and obtain a good agreement for $\alpha$ and $\beta$ (Fig. 1). We calculate the value of $G$ from our simulations as shown in Fig. 2. It is noticed that the numerical results indicate the spin injection efficiencies are somewhat lower for higher values of $P$, as the analytical expression (4) is an approximation in this region. The good agreement confirms that the space charge effects are absent in this case.

\section{B. Violated Charge Neutrality}

Next, we modify the doping in the ferromagnet by changing the ratio $K_{1}$ to violate the charge neutrality. One can introduce an accumulated $\left(K_{1}>1\right)$, charge neutral $\left(K_{1}=1\right.$, as already mentioned) or even a depleted $\left(K_{1}<1\right)$ charge

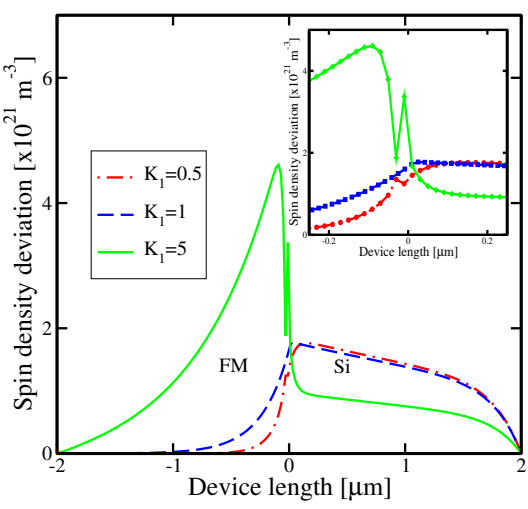

Fig. 3. Spin density accumulation over the bar, when the charge current density is fixed to $23.4 \mathrm{MA} / \mathrm{m}^{2} . P=0.2 . K_{1}$ is the doping ratio $(F M / S i)$. The inset figure is highlighting the interface region.

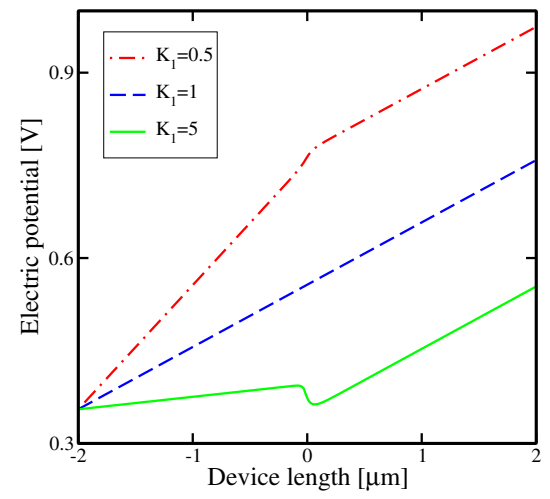

Fig. 4. Electric potential lines through the bar, under similar conditions as in Fig. 3.

(and hence spin) injection source. Charge injection to $\mathrm{Si}\left(K_{1}>1\right)$ or charge release $\left(K_{1}<1\right)$ always cause a non-zero charge current through the junction, even at the absence of an external electric field. This charge current introduces spin accumulation/depletion in $\mathrm{Si}$. We investigate the carrier distribution and the spin current variation along the bar considering a fixed charge current and we fix $J_{c}$ to be $23.4 \mathrm{MA} / \mathrm{m}^{2}$. It is noted that the spin density ( $s$, and hence the spin current $J_{s}$ ) behaves differenetly at the interface and in the bulk. When $K_{1}>1\left(K_{1}<1\right), s$ gradually piles up (drops down) in the bulk $F M$ and drops down (piles up) in the bulk Si (Fig. 3), compared to what we get at the charge neutrality condition. This phenomenon happens due to the difference in the material conductivity (proportional to the doping 


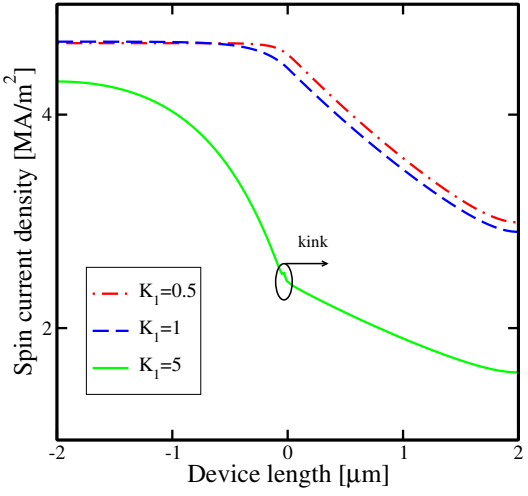

Fig. 5. Spin current density through the interface and the bulk, with the same conditions as in Fig. 3. A small amount of enhancement is noticed at the interface, when $K_{1}=5$.

concentration), and the bulk electric field, which eventually modifies the effective spin diffusion length. On the contrary, when $K_{1}>1\left(K_{1}<1\right), s$ develops a dip (peak) at the $F M$ interface followed by a sharp peak (dip) at the semiconductor (Fig. 3 inset) interface. These features are correlated with the charge depletion (accumulation) at the ferromagnetic/nonmagnetic interface, which result in the formation of the potential profile with a barrier for electrons (Fig. 4). These interface effects give rise to a small alteration in the spin current (Fig. 5) at the interface, however persisting only up to the charge screening length $\left(\lambda_{D}\right.$, typically $40 \mathrm{~nm}$ ). Now, we look for the spin injection efficiencies in $\mathrm{Si}$ taken at a distance $\lambda_{D}$ away from the interface $\left(\alpha_{D}\right.$, and $\beta_{D}$ respectively). Fig. 6 shows an increment in both $\alpha_{D}$, and $\beta_{D}$, compared to the charge neutrality condition, if and only if the spins are injected into Si from a charge depleted source. The values are limited by $F M$ bulk spin polarization.

\section{CONCLUSION}

Spin injection in a semiconductor structure from a ferromagnetic material is delineated. At charge neutrality, the spin injection efficiency in the $\mathrm{Si}$ side is determined by the bulk spin polarization at the magnetic side and the charge current. If a constant charge current is maintained with violated charge neutrality under a fixed spin polarization, one observes an increase or decrease of the spin current near the interface region, however persisting up to the screening length. Under

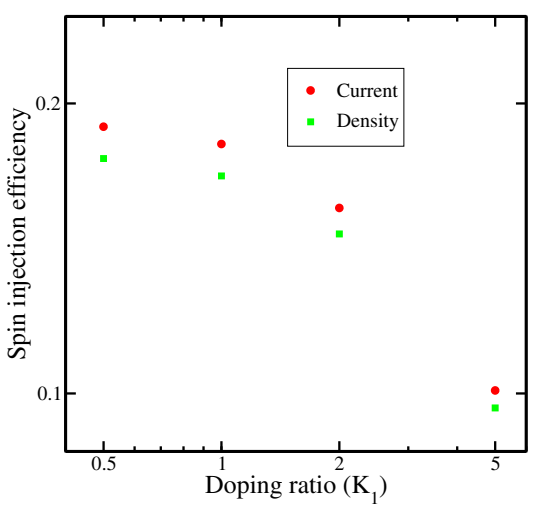

Fig. 6. Spin density and spin current injection efficiencies $\left(\alpha_{D}\right.$, and $\left.\beta_{D}\right)$, taken at $\lambda_{D}$ away from the interface and inside $\mathrm{Si}$, with the same conditions as in Fig. 3. $P=0.2$ is kept.

similar conditions, the spin injection efficiency in the semiconductor bulk severely decreases, if the spins are injected from a highly charge accumulated source. The efficiency can be increased, if spins are injected from a charge depleted source, but its value is always limited by the bulk spin polarization of the ferromagnetic material.

\section{ACKNOWLEDGMENT}

This work is supported by the European Research Council through the grant \#247056 MOSILSPIN.

\section{REFERENCES}

[1] D. E. Nikonov, and I. A. Young, "Overview of BeyondCMOS Devices and a Uniform Methodology for Their Benchmarking", cond-mat.mes-hall, vol. 36, 2013.

[2] R. Jansen, "Silicon Spintronics," Nature Materials, pp. 120, 2012.

[3] Y. Song, and H. Dery, "Magnetic Field Modulated Resonant Tunneling in Ferromagnetic-Insulator-Nonmagnetic Junctions," arXiv/1401.7649, 2014.

[4] M.R. Sears, and W.M. Saslow, "Spin Accumulation at Ferromagnet/Nonmagnetic Material Interfaces", Phys. Rev. B, vol. 85, no. 014404, pp. 1-10, 2012.

[5] J. Ghosh, V. Sverdlov, T. Windbacher, and S. Selberherr, "Spin Injection and Diffusion in Silicon Based Devices from a Space Charge Layer", J. Appl. Phys., vol. 115, no. 17C503, pp. 1-3, 2014.

[6] Z.G. Yu, and M.E. Flatte, "Spin Diffusion and Injection in Semiconductor Structures: Electric Field effects", Phys. Rev. B, vol. 66, no. 235302, pp. 6-9, 2002.

[7] Y. Song, and H. Dery, "Spin Transport Theory in Ferromagnet/Semiconductor Systems with Noncollinear Magnetization Configurations", Phys. Rev. B, vol. 81, no. $045321,2010$.

[8] D. Scharfetter, and H. Gummel, "Large-Signal Analysis of a Silicon Read Diode Oscillator", Electron Devices, IEEE Transactions on, vol. 16, issn. 0018-9383, 1969. 